\title{
Oriented Polar Snakes for Phase Contrast Cell Images Segmentation
}

\author{
Mitchel Alioscha-Perez ${ }^{1}$, Ronnie Willaert ${ }^{2}$, Helene Tournu $^{3,4}$, Patrick Van Dijck ${ }^{3,4}$ \\ and Hichem Sahli ${ }^{1,5}$ \\ 1 Dept. Electronics \& Informatics (ETRO), Vrije Universiteit Brussel (VUB), Belgium \\ 2 Research Group Structural Biology Brussels (SBB), Vrije Universiteit Brussel (VUB), \\ Belgium \\ 3 Department of Molecular Microbiology, VIB, KU Leuven \\ ${ }^{4}$ Laboratory of Molecular Cell Biology (MCB), KU Leuven, Leuven, Belgium \\ ${ }^{5}$ Interuniversity Microelectronics Center (IMEC), Leuven, Belgium
}

\begin{abstract}
Noninvasive imaging of unstained living cells allows to study living specimens without altering them, and is a widely used technique in biotechnology for determining biological and biochemical roles of proteins. Fluorescence and contrast images are both used complementarily for better outcomes. However, segmentation of contrast images is particularly difficult due to the presence of lighting/shade-off artifacts, defocused scans, or overlapping. In this work, we make use of the optical properties intervening during the image formation process for cell segmentation. We propose the shear oriented polar snakes, an active contour model that implicitly involves phase information. Experimental results confirms the method suitability for cell images segmentation.
\end{abstract}

Keywords: active contours, image phase estimation, smart markers, image segmentation.

\section{Introduction}

Determining biological and biochemical roles of proteins is one of the critical tasks in biotechnology. Towards this goal, one of the most popular and successful technique is the noninvasive imaging of unstained living cells, which is mostly based on fluorescence and contrast imaging, and allows to study living specimens without altering them. Very often, fluorescence images with expression of (targeted) proteins and contrast images with individual cells information, are both used complementarily. Their combination provides better ways to measure the number of individual cells in populations, better sub-cellular localization of proteins, more precise individual cellular morphology measurements, cells population time-lapse evolution analysis, among other related tasks.

Considering images such as the one illustrated in Fig.11a), several approaches could be considered for segmentation, such as mathematical morphology [1], Level-sets [6] and active contours [7]. Authors in [4] estimate (restore) the phase information related to the image in question, and perform a subsequent level-set segmentation over the artifacts-free restored phase image, obtaining better results than segmenting the original phase contrast image. They showed how the resultant estimated image can be better segmented, even when using simple automatic thresholding techniques. Despite the 
mentioned improvements several drawbacks are still present. They use a more general/complex formulation for the phase estimation, but convexity on the related optimization problem can not be granted. Also, their strategy fails to cope with high-density overlapping (multilayer) cells cultures, where superposition of lighting/shade-off of individual cells introduces errors in the recovered phase (see Fig 1). As consequence, these errors have a high negative impact on the level-set segmentation since they use the estimated phase for the initialization of the level-set and for the segmentation as well.

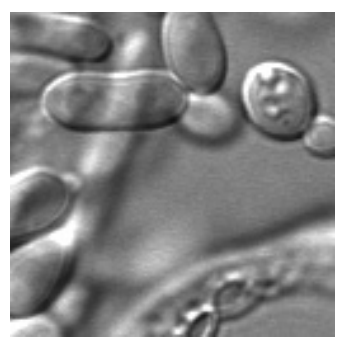

(a) Phase Contrast Image.

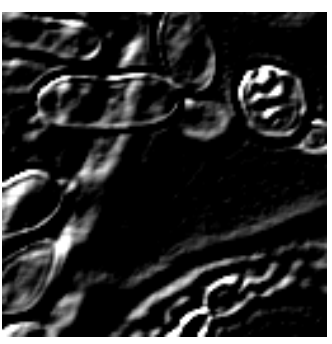

(b) Estimated Phase.

Fig. 1. Errors on the estimated phase are caused by the superposition of cells in multilayer cultures

Inspired by the work of Yin et al. [4], we estimate the phase using a simple and well known formulation that grants convergence towards the approximation, and we use a parametric active contour (snakes) with a novel energy functional.

In this paper we propose the shear oriented polar snakes [8], a parametric active contour model that: i) implicitly involves phase information on its energy functional using a provided shear-angle orientation, and ii) use the estimated phase image for the snakes initialization, but minimize the energy on the original image. The segmentation process is illustrated in Fig.(2).

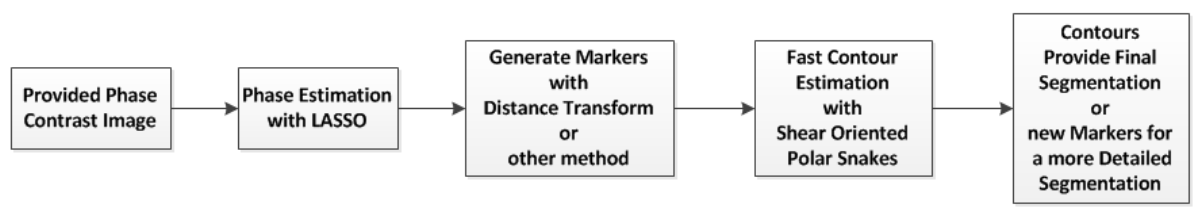

Fig. 2. The proposed segmentation process

The reminder of this paper is organized as follows: a review of phase-contrast optics and active contours models is provided in Section 2, along with the proposed energy model details. Experimental results are reported and discussed in Section 3, while conclusions are given in Section 4. 


\section{Oriented Polar Snakes}

\subsection{Phase Contrast Optics}

Phase contrast imaging is used to image phase objects, which are almost transparent objects but with a refractive index different from their surrounding medium. The different optical path length, obtained from two mutually orthogonal light beams shifted by a vector $\tau(m, \theta)$, is transformed into intensity variations.

Let's consider a Point Spread Function (PSF) that represent the finite response of a focused optical system. Considering diffraction due to the microscope optics, a discrete PSF can be expressed as:

$$
f(x, y)=(-x \cos \theta-y \sin \theta) \exp \left(-\frac{\left(x^{2}+y^{2}\right)}{\sigma^{2}}\right) \quad \forall x, y \in[-M, M]
$$

which leads to the image model $I$ involving the PSF and the artifact-free phase image $\varphi$, as follows:

$$
I(x, y)=\varphi(x, y) \otimes f(x, y)+\eta(x, y) .
$$

being $\otimes$ a convolution operator, and $\eta($.$) an additive noise function of unknown distri-$ bution.

The phase reconstruction problem consist in finding $\varphi$ from an observed $I$ (inverse problem). Despite of the simplicity of Eq. (2), its solution is seldom straightforward. Direct deconvolution is not possible to be performed due to the sensitiveness to noise.

One of the most popular ideas considers to find a $\varphi$ such that $\varphi \otimes f$ that is as close as possible to $I$, and use the squared residuals as penalization, named least squares. Since a regularization of $\varphi$ is preferable, the least absolute shrinkage and selection operator (LASSO) offers an attractive formulation [9]:

$$
\min _{\varphi}\|I-\varphi \otimes f\|^{2}+\lambda\|\varphi\|_{1}
$$

The main benefit of this formulation is that it enforce sparsity on the solution due to the $\ell_{1}$-norm regularization, while keeping the optimization problem convex. In few words, it enforce the recovered phase to have as few pixels as possible.

\subsection{Active Contour Models}

The two main categories of active contours can be used: geometric and parametric snakes. In geometric snakes, the curve is described from a level set representation, while in parametric snakes it is described as a discrete collection of points. It is known that parametric snakes are much faster than geometric snakes, which support our model selection.

Let's consider the generic family of curves $\mathcal{C}_{q}$ depending on a $n$-dimensional parameter vector $q$ and defined in the image plane by: 


$$
\mathcal{C}_{q}: x(u)=x_{c}+r_{q}(u)\left(\begin{array}{c}
\sin u \\
\cos u
\end{array}\right)
$$

where $r_{q}(u)$ is the radius function depending on $u \in(0,2 \pi)$, and $x_{c}$ is a point inside the contour. Then, the total active contour energy can be defined as:

$$
E_{a c}=\alpha \int_{0}^{2 \pi}\left|\frac{\partial \mathcal{C}_{q}}{\partial u}\right|^{2} d u+\beta \int_{0}^{2 \pi}\left|\frac{\partial^{2} \mathcal{C}_{q}}{\partial^{2} u}\right|^{2} d u+\int_{0}^{2 \pi} E_{i m}\left(\mathcal{C}_{q}\right) d u
$$

The first two terms, named potential energy or internal energy, accounts for elasticity (or extent) and curvature, respectively. The third term, the image energy, provides a cost evaluation of the contour according to the image. This terms is discretized as:

$$
\int_{0}^{2 \pi} E_{i m}\left(\mathcal{C}_{q}\right) d u=\sum_{k=0}^{N-1} E_{i m}\left(\mathcal{C}_{q}, u_{k}\right)
$$

being $u_{k}=\frac{2 \pi}{N} k$ (direction of $k$-th element). The external (image) energy can be defined in several ways, depending on the characteristics of the problem in question.

\subsection{Energy Functional}

In this work we propose the following external (image) energy functional:

$$
E_{i m}\left(\mathcal{C}_{q}, u_{k}\right)=\sin ^{2}\left(u_{k}-\theta\right)\left|I_{\mu}\left(C_{q}\left(u_{k}\right)\right)\right|-\cos \left(u_{k}-\theta\right) I_{\mu}\left(C_{q}\left(u_{k}\right)\right)-\left|\nabla I\left(C_{q}\left(u_{k}\right)\right)\right|
$$

with $\mu$ the intensity mean, $I_{\mu}()=.I()-.\mu, \nabla I($.$) the intensity gradient, and \theta$ the provided shear (angle) direction information.

The proposed energy enforce low energy values for $E_{i m}$ whenever a lighting/shadeoff effect is found in a parallel direction with respect to the provided shear angle, decreased even more if this match with a high gradient value.

\subsection{Numerical Implementation}

We opted for using a dynamic programming approach [2] to solve Eq.(5) based on the Bellman's Principle of Optimality [5], with a discrete-time finite horizon in a onedimensional formulation (states related only to $r_{q}$ ). Since the initial number of markers can be considerable big, the use of a lower number of snakes elements (usually between $N=45$ and $N=90$ ) allowed us to find optimal solutions relatively fast. Depending on the parameters $\alpha$ and $\beta$, the execution time varies between 260 and 860 milliseconds per snake, using a Matlab implementation of the chosen numerical method. It is also worth noting that each individual snake can be optimized in parallel. 


\subsection{Segmentation}

After the artifacts-free phase image has been estimated (see Fig.1 (b)), a distance transform is applied on it; then a local maxima points detection provides the set of initial markers, one point per marker. The set of automatically generated markers will be used for the initialization of the active contours (see Fig. 3a) as follows: each marker correspond to exactly one snake, that initially starts with radius $r_{q}()=$.1 and centroid $x_{c}$ on the corresponding marker. Once the snake models has been initialized, the next step is to optimize them by minimizing the active contour energy Eq. 5.

After all the snakes has been optimized, we expect that for initial markers located on the same region, their corresponding snakes converges towards the same boundaries. Then, likewise in [3], we perform a contour evaluation consisting in discarding those contours (and markers) overlapping in a high percent with another contour of higher priority, where the priority is given by evaluating the contour using Eq.(7).

\section{Experimental Results}

\subsection{Qualitative Analysis}

In order to highlight the benefits of the proposed energy functional of Eq. (7) with respect to the standard gradient-based $E_{i m}\left(C_{q}, u_{k}\right)=-\left|\nabla I\left(C_{q}\left(u_{k}\right)\right)\right|$, we used a simulated phase contrast image of two overlapping cells, and a real phase contrast image fragment where a cell is out of the focal plane.

The proposed model can find the appropriate contours (see Fig $3 \mathrm{r}$ ), even without assuming any curvature in the contour $(\beta \approx 0$ in Eq. 5), while the gradient-based energy model fail to do so (see Fig $3 \mathrm{~b}$ ), requiring certain specific values of curvature parameter to find the appropriate contour.

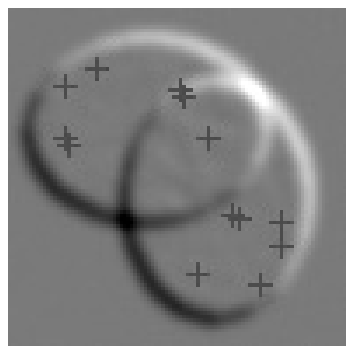

(a) Automatically generated initial markers.

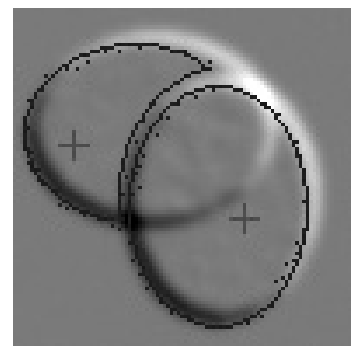

(b) Gradient-based energy contours.

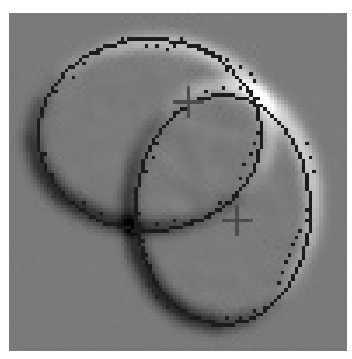

(c) Proposed energy contours.

Fig. 3. The proposed energy estimates the contours better than a standard gradient-based energy, even without assuming any curvature in the contour

We also show how the gradient-based energy model is very sensitive to the lighting/shade-off artifact, and the contour solution takes the extreme values of the gradient, resulting in a contour shifted in the shear-angle direction. The proposed model 
can better cope with this problem as can be seen in Fig.(4), where the most sensitive snake elements of our proposed solution are those orthogonal to the shear-angle direction.

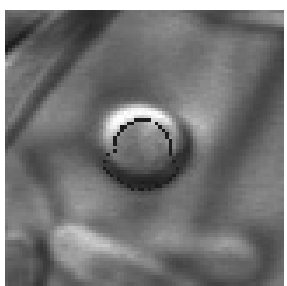

(a) Gradient-based energy contour.

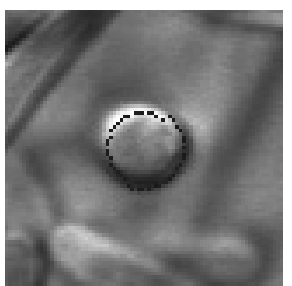

(b) Proposed energy contour.

Fig. 4. The proposed energy can better cope with lighting/shade-off artifacts

The proposed model can also deal properly with corners and broken lines, as shown in Fig.(5). In this simulated image with corners and shear angle $\theta=\frac{\pi}{4}$, the proposed energy prevented each contour's convergence to stop on the boundaries of the other rectangle, by enforcing high energy values (penalization) on dark boundaries located to the right of marker A; similarly on bright boundaries located to the left of marker B.
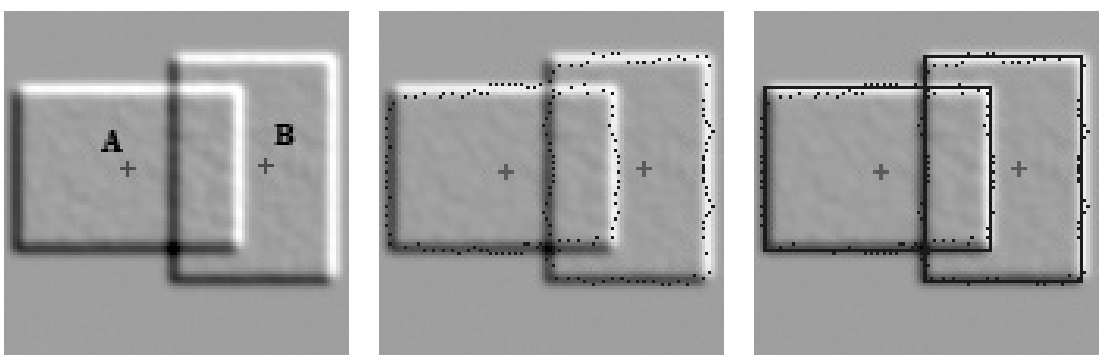

Fig. 5. The proposed energy can cope with other type of objects

\subsection{Real Specimen Images}

For the experiments on real specimen images, serum-induced cultures of the fungus Candida albicans were used in this study in order to obtain mixed populations of different cell morphologies of the fungus. Cells expressing two protein fusions to a monomeric GFP variant are displayed. Genomic copy tagging of HSP90 and RAS2 loci were performed in wild type strain SC5314. Cells were grown at $37^{\circ} \mathrm{C}$ for three hours and images were acquired by confocal microscopy (Olympus Fluoview ${ }^{\mathrm{TM}}$ ). Fluorescence images were obtained with Alexa Fluor 488 excitation laser. 
The figures 6(a) and (e) depict the obtained images. As expected, the number of automatically generated markers (Fig. 6(c) and (g)) from the estimated phase (Fig. 6(b) and (f)) was higher than the number of cells in the image. The fact that most of the markers fall inside cell regions allowed the active contours to converge towards individual cell boundaries. As illustrated in Fig. (6) (d) and (h)), the proposed contour evaluation process provides almost one contour/marker per individual cell in the image.

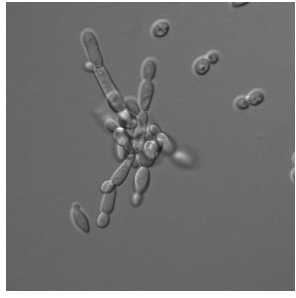

(a) Contrast Image.

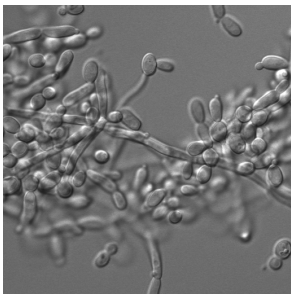

(e) Contrast Image.

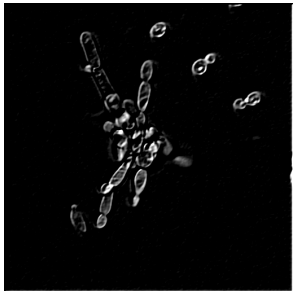

(b) Estimated Phase.

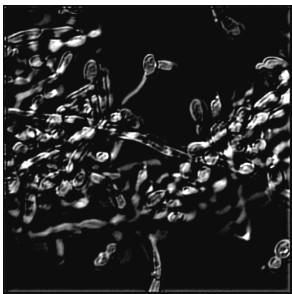

(f) Estimated Phase.

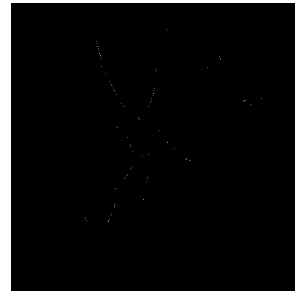

(c) Generated Markers.

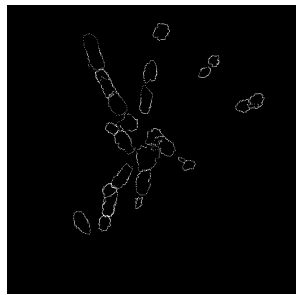

(d) Contour Approximation.

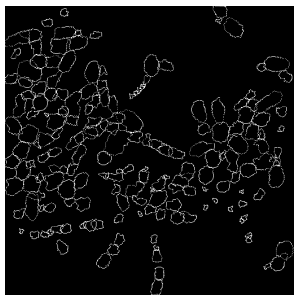

(g) Generated Markers. (h) Contour Approximation.

Fig. 6. The estimated phase provides the basis to generate markers for snakes initialization

To assess the effectiveness of the proposed Oriented Polar Snakes, we compared it to the markers-controlled watershed segmentation. Both segmentation schemes have been initialized using exactly the same set of markers. As it can be seen from Fig 7 , the proposed method produces well segmented individual cells compared to the oversegmented watershed. It has to be noted that, subsequent region merging of the obtained watershed segments is difficult due to the type of contrast image.

\section{Conclusions}

In this work we presented a segmentation method, based on an active contour model with a novel energy functional, that takes into account the nature of this type of images. The method has been developed specifically for contrast microscopy cells images, but can be easily extended and applied to any other type of phase contrast images. In future works we will perform a quantitative analysis of the method over different datasets of phase contrast images. We will also investigate different ways to enhance the initial marker selection in order to decrease the initial number of snakes. 


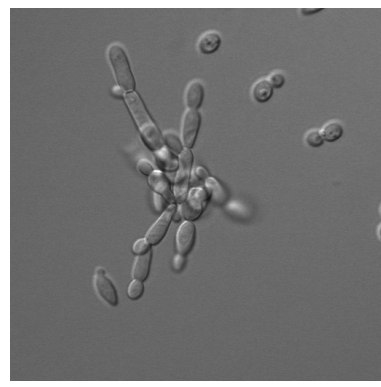

(a) Original Contrast Image.

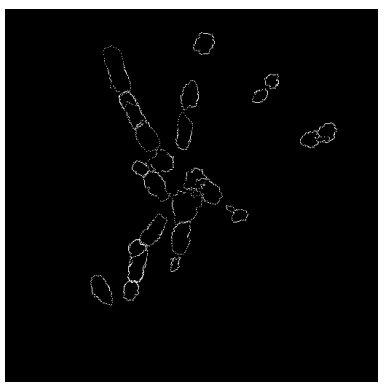

(b) Proposed Segmentation.

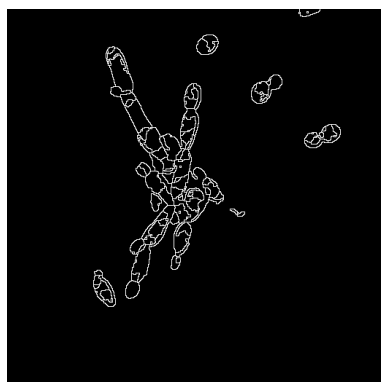

(c) Watershed Segmentation.

Fig. 7. The proposed segmentation provides more accurate individual region/cells than watershed segmentation, using the same markers for initialization

Acknowledgments. The Belgian Federal Science Policy Office (Belspo) and the European Space Agency (ESA) PRODEX program supported this work.

\section{References}

1. Koyuncu, C.F., Arslan, S., Durmaz, I., Cetin-Atalay, R., Gunduz-Demir, C.: Smart Markers for Watershed-Based Cell Segmentation. PLOS ONE 7(11), e48664 (2012), doi:10.1371/journal.pone.0048664

2. Cohen, L.D., Kimel, R.: Global minimum for active contour models: A path approach. Int. Jour. Comp. Vis. 24, 57-78 (1997)

3. Wienert, S., Heim, D., Saeger, K., Stenzinger, A., Beil, M., Hunfnagl, P., Dietel, M., Denkert, C., Klauschen, F.: Detection and segmentation of cell nuclei in virtual microscopy images: a minimum-model approach. Scientific Reports 2, 503-510 (2012)

4. Yin, Z., Kanade, T., Chen, M.: Understanding the phase contrast optics to restore artifact-free microscopy images for segmentation. Medical Image Analysis 16, 1047-1062 (2012)

5. Bellman, R., Dreyfus, S.: Applied Dynamic Programming. Princeton University Press, Princeton (1962)

6. Ambühl, M., Brepsant, C., Meister, J., Verkhovsky, A., Sbalzarini, I.: High-resolution cell outline segmentation and tracking from phase-contrast microscopy images. J. Microsc. 245(2), 161-170 (2012)

7. Seroussi, I., Veikherman, D., Ofer, N., Yehudai-Resheff, S., Keren, K.: Segmentation and tracking of live cells in phase-contrast images using directional gradient vector flow for snakes. J. Microsc. 247(2), 137-146 (2012)

8. Collewet, C.: Polar snakes: A fast and robust parametric active contour model. In: IEEE Int. Conf. on Image Processing (ICIP), pp. 3013-3016 (2009)

9. Tibshirani, R.: Regression Shrinkage and Selection via the Lasso. J. R. Static. Soc. 58(1), 267-288 (1996) 